\title{
Plan de desarrollo humano nicaragüiense, 2012-2016: un enfoque para la educación y desarrollo rural
}

\section{Plan de desarrollo humano nicaragüense, 2012-2016: un enfoque para la educación y desarrollo rural}

\author{
Martínez Andrade, Erick; Rivas García, Julio Antonio; Villalobos \\ Maradiaga, Edixia María; Gómez Trujillo, Elvin Ariel; Zúniga \\ González, Carlos Alberto; Editor Academico Dr. Angel Sol Sanchez
}

\author{
Erick Martínez Andrade \\ eandrade27@gmail.com \\ Centro Universitario Regional (CUR-Somotillo). \\ UNAN-León, Nicaragua \\ Julio Antonio Rivas García \\ eandrade27@gmail.com \\ Centro Universitario Regional (CUR-Somotillo). \\ UNAN-Le, Nicaragua \\ Edixia María Villalobos Maradiaga \\ eandrade27@gmail.com \\ Centro Universitario Regional (CUR-Somotillo). \\ UNAN-León., Nicaragua \\ Elvin Ariel Gómez Trujillo \\ eandrade27@gmail.com \\ Centro Universitario Regional (CUR-Somotillo). \\ UNAN-León., Nicaragua \\ (D) Carlos Alberto Zúniga González \\ czuniga@ct.unanleon.edu.ni \\ Departamento de Agroecología. CICAEA UNAN- \\ León., Nicaragua \\ Editor Academico Dr. Angel Sol Sanchez \\ Colegio de Posgraduados Mexico, México
}

Revista Iberoamericana de Bioeconomía y Cambio Climático

Universidad Nacional Autónoma de Nicaragua, León, Nicaragua ISSN-e: 2410-7980

Periodicidad: Semestral

vol. 2, núm. 1, 2016

czuniga@ct.unanleon.edu.ni

Recepción: 30 Octubre 2015

Aprobación: 04 Mayo 2016

URL: http://portal.amelica.org/ameli/journal/394/3941750027/

DOI: https://doi.org/10.5377/ribcc.v2i1.5707

Autor de correspondencia: eandrade27@gmail.com
Resumen: El presente estudio analizó las bondades del Plan Nacional de Desarrollo Humano (PNDH) 2012 - 2016, formulado y aplicado en Nicaragua y rectorado por el gobierno de Reconciliación y Unidad Nacional (GRUN), como un instrumento de la Educación y el Desarrollo Rural para llevar a la práctica las políticas públicas, estrategias y acciones de Nación, que permitan alcanzar los propósitos de reducción de la pobreza, Desarrollo Humano, Social, Económico, con justicia y desde el protagonismo de la persona, las familias y la comunidad. El PNDH es un plan vivo, en continua construcción, abierto a los aportes de la sociedad nicaragüense, que es actualizado de manera periódica para ajustarse a los procesos cambiantes internos y externos, es un plan Maestro que sirve de referencia para el mediano y largo plazo, es decir cualquier política pública tendrá como referencia la articulación con este plan en el cual se aspira a tener una estructura holística e integral. En el marco de la Educación y Desarrollo Rural el estudio del PNDH resulta ser un instrumento para mejorar la calidad de vida de los y las nicaragüenses ubicándolos como centro y sujeto primordial del desarrollo, por medio del crecimiento económico con equidad social y la transformación de los métodos de producción y de los patrones de consumo y que se sustenta en el equilibrio ecológico y el soporte vital de la región.

Palabras clave: Cohesión Social, Equilibrio Ecológico, Crecimiento económico, Equidad Social, Pobreza, Gobierno, Integración, Seguridad, Solidaridad, Desarrollo Humano.

Abstract: This study analyzed the benefits of the National Human Development Plan (PNDH) 2012-2016, formulated and applied in Nicaragua and governed by the Government of Reconciliation and National Unity (GRUN), as an instrument of Education and Rural Development to bring to put into practice the public policies, strategies and actions of the Nation, which allow achieving the purposes of poverty reduction, Human, Social, Economic Development, with justice and from the role of the person, families and the community. The $\mathrm{PNDH}$ is a living plan, in continuous construction, open to the contributions of Nicaraguan society, which is periodically updated to adjust to changing internal and external processes, it is a Master plan that serves as a reference for the medium and long term , that is to say, any public policy will have as 
reference the articulation with this plan in which it aspires to have a holistic and integral structure. Within the framework of Education and Rural Development, the study of the PNDH turns out to be an instrument to improve the quality of life of Nicaraguans, placing them as the center and primary subject of development, through economic growth with social equity and the transformation of production methods and consumption patterns and that is based on the ecological balance and vital support of the region..

Keywords: Social Cohesion, Ecological Balance, Economic growth, Social Equity, Poverty, Government, Integration, Security, Solidarity, Human development.

\section{INTRODUCCIÓN}

El Plan Nacional de Desarrollo Humano es un proyecto que incluye desde poner en órbita un satélite exclusivo para Nicaragua, construcción de nuevos aeropuertos, muelles en las costas, transformar la matriz energética a energía renovable y apoyar a las familias que trabajan por cuenta propia. Un avance que con los programas que se han ejecutado del 2007 a la fecha (GRUN: 2011, 2012, 2013).

El GRUN apuesta en acompañamiento con el consenso, relaciones respetuosas internas y externas, no en busca de la unipolaridad, sino una multipolaridad en donde diferentes culturales, miembros de la sociedad puedan convivir en paz, en la lucha por la restitución de los derechos de los y las nicaragüenses.

La seguridad, la lucha contra el crimen organizado, los comités de prevención del delito todo ello contemplado en el PNDH en busca de la reconstrucción de la sociedad.

Nuestra costa Caribe es pilar fundamental del PNDH en donde se contempla el respeto a la multiculturalidad, la autonomía de nuestras regiones autónomas y que está incluida en los procesos que reformas constitucionales que el gobierno realizara en este año 2013 para profundizar la restitución de derechos a los pueblos de nuestra costa atlántica que quedara plasmado por ley que pasara a llamarse "COSTA CARIBE NICARAGUENSE".

Un aspecto muy importante es que se toma en cuenta el acceso a la ciencia y la tecnología en donde se hace hincapié en un acceso pertinente, es decir no a cualquiera información se tendrá acceso, sino de uso de acuerdo a las realidades de nuestra nación. Roustan-Espinosa, I. M., Quezada, F., \& Huete-Pérez, J. A. (2006) hizo un análisis de esta temática enfatizando la Biotecnología como un requerimiento tecnológico emergente.

Lineamientos estratégicos que describen la estrategia en este plan: participación ciudadana. Gobernabilidad democrática, transparencia y probidad, seguridad ciudadana, acceso y calidad en la impartición de la justicia, fortalecimiento del estado y capacitación de la administración pública responsable, armonización de los poderes del Estado, descentralización y fortalecimiento municipal, y seguridad al derecho de propiedad, se contempla también la tasa de escolaridad preescolar, primaria y secundaria (GRUN: 2011, 2012, 2013).

NotAS DE AUTOR

eandrade27@gmail.com 


\section{DESARROLLO}

Los estudios realizados y plasmados en el plan confirman la importancia de continuar apoyando inversiones en el área rural, donde existen brechas de cobertura y desafíos en materia de infraestructura y servicios en ambos sectores.

Cabe mencionar que uno de los sectores económicos en que Nicaragua tiene una ventaja comparativa y bastantes oportunidades es en el sector agropecuario, en el cual estaremos trabajando para desarrollar cadenas de valor.

La Estrategia del PNDH está construida en base a doce lineamientos que ponen de manifiesto la Teoría General del Desarrollo Sostenible (GRUN: 2011, 2012, 2013), además de combinar la continuidad de las políticas que han transformado al país a partir del 2007, con la incorporación de nuevas prioridades cuyo propósito es atender las necesidades inaplazables de transformación productiva y social del país, detalle de estos lineamientos a continuación:

1. EL CRECIMIENTO ECONÓMICO Y LA ESTABILIDAD MACROECONÓMICA CON INCREMENTO DEL TRABAJO Y REDUCCIÓN DE LA POBREZA.

El objetivo de la política económica continuará siendo preservar un marco macroeconómico estable que estimule la inversión privada nacional y extranjera, la inversión pública, el espíritu emprendedor, la producción y la productividad, a fin de generar mayor crecimiento económico con mayores beneficios sociales para los más pobres y reducción de las desigualdades.

2. FORTALECIMIENTO DE LA GRAN ALIANZA ENTRE LOS TRABAJADORES, LOS PRODUCTORES Y EL GOBIERNO

Una característica que ha distinguido a este gobierno, ha sido su capacidad para conciliar las posiciones más divergentes, fortaleciendo la Gran Alianza Nacional de Trabajadores de la Ciudad y el Campo, Productores, Empresarios, Cooperativas, Pequeña, Mediana y Gran Industria y el Gobierno Sandinista de Reconciliación y Unidad Nacional, con el acompañamiento de la Cooperación Internacional y las inversiones nacionales y extranjeras, logramos alcanzar, todos unidos, un crecimiento en nuestra economía. (Arrien, J. 2006)

\section{POLÍTICA EXTERIOR Y DE COOPERACIÓN EXTERNA SOBERANA,} INDEPENDIENTE Y ABIERTA A TODOS LOS PAÍSES DEL MUNDO EN EL COMBATE A LA POBREZA EN BENEFICIO DE LAS FAMILIAS NICARAGÜENSES

La política exterior y de cooperación externa del Gobierno se ha implementado bajo los principios de independencia, soberanía, autodeterminación, dignidad, respeto, unidad y solidaridad con todos los países y organismos internacionales con los que Nicaragua sostiene relaciones. El Gobierno promueve las relaciones internacionales de Nicaragua con todos los pueblos y gobiernos del mundo a nivel bilateral y multilateral, en el marco de la Constitución y el Derecho Internacional.

4. INTEGRACIÓN CENTROAMERICANA, UNIÓN DEL ALBA Y ARTICULACIÓN LATINOAMERICANA Y CARIBEÑA A TRAVÉS DE LA CELAC

Nuestro Gobierno ha seguido los principios contenidos en la Constitución Política, que establecen la posición de Nicaragua frente a la integración.

El GRUN apoya y promueve todos los esfuerzos del Sistema de la Integración Centroamericana (SICA) para lograr la integración política, económica y de cooperación en América Central, así como los esfuerzos por establecer la paz en la región. (Agosin, M.R y Machado R. 2004)

\section{SEGURIDAD CIUDADANA Y LUCHA CONTRA EL NARCOTRÁFICO Y EL CRIMEN ORGANIZADO}

Se ha aplicado la estrategia de seguridad preventiva y proactiva a través del Modelo de Seguridad Comunitario, que prioriza la seguridad de las personas, las familias y las comunidades en el campo y la ciudad.

Los objetivos estratégicos están orientados a desarrollar y fortalecer los programas de Gobernabilidad, lucha contra el crimen organizado, reinserción social en el Sistema Penitenciario Nacional, garantizar la 
efectividad de los servicios de protección a las personas y sus bienes, incrementar los niveles de seguridad en los puestos fronterizos, mejorar la detección de ilícitos y fortalecer la efectividad de los servicios migratorios.

6. DESARROLLO INTEGRAL DE LA COSTA CARIBE.

El desarrollo integral de la costa Caribe constituye uno de los pilares más importantes de la estrategia de reducción de la pobreza en Nicaragua.

La premisa fundamental de la Estrategia de Desarrollo de la Costa Caribe (EDCC) es que el desarrollo surge desde adentro, siendo su centro el ser humano, los valores ancestrales y solidarios, la participación comunitaria, aspectos que promueven que las personas, las familias, las comunidades y las instituciones asuman el desarrollo como propio, en corresponsabilidad, construyendo el buen vivir y el bien común.

\section{GESTIÓN PÚBLICA PARTICIPATIVA Y DEMOCRACIA DIRECTA}

Los avances en la reducción de la pobreza y la desigualdad en Nicaragua han sido posibles por las prácticas de gestión pública con la participación del pueblo, ejerciendo con protagonismo la democracia directa.

\section{EL BIEN COMÚN Y LA EQUIDAD SOCIAL DE LAS FAMILIAS NICARAGÜENSES}

El Gobierno de Reconciliación y Unidad Nacional ha venido desarrollando una política social desde la restitución de derechos, recuperación de valores y fortalecimiento de capacidades en favor de las familias pobres, generando resultados positivos como la reducción de la pobreza y la desigualdad. (Borraz, F y Gonzáles N. 2008)

9. CIENCIA, TECNOLOGÍA, INNOVACIÓN Y EMPRENDEDURISMO PARA LA TRANSFORMACION DE NICARAGUA (CTIE).

La transformación de Nicaragua sólo será posible con la transformación de las y los nicaragüenses, y una de las vías para lograrlo es a través de la ciencia, tecnología, innovación y emprendedurismo (CTIE).

El objetivo de esta política es impulsar el salto cualitativo hacia mayores niveles de productividad, de valor agregado, de trabajadores con mayor calificación, de trabajo de mayor calidad, de mayor competitividad de las pymes y todas las empresas, de la construcción del desarrollo humano y del desarrollo del país.

Nicaragua tiene el propósito de superar las brechas entre las necesidades actuales y futuras de los sectores económicos y sociales, y los objetivos, alcances y planes de la investigación científica que se desarrolla en el país.

10. EL SECTOR PRODUCTIVO PRIORIZANDO LA ECONOMÍA FAMILIAR, COMUNITARIA Y COOPERATIVA, Y LA SOBERANÍA Y SEGURIDAD ALIMENTARIA, EN UN CONTEXTO DE CAMBIO CLIMÁTICO

El GRUN definió una estrategia productiva que prioriza el fortalecimiento de la economía familiar, comunitaria, cooperativa y asociativa, que tiene como objetivos reducir la pobreza y desigualdad, aumentar la producción y la productividad, fortalecer la seguridad y soberanía alimentaria, y adaptar el país al cambio climático.

La política de apoyo a los pequeños productores ha sido integral, capitalizando y fortaleciendo las capacidades productivas de las familias pobres, con capacitación y asistencia técnica, créditos, insumos, y apoyo en las diferentes formas asociativas incluyendo las cooperativas, en busca del auto sustento familiar, lo que está contribuyendo activamente al aumento de la disponibilidad de alimentos en los hogares de las familias pobres y la reducción de la pobreza.

11. INFRAESTRUCTURA SOCIAL, PRODUCTIVA, ENERGÉTICA, DE TRANSPORTE Y TURÍSTICA PARA LA TRANSFORMACIÓN DE NICARAGUA

Se ha planteado una nueva estrategia para el desarrollo de la infraestructura social, productiva, energética, de transporte, puertos y aeropuertos, telecomunicaciones y turismo, con el propósito de elevar la eficiencia, el rendimiento e impacto de la producción nacional, reducir la alta dependencia del país a elementos importados, incluyendo hidrocarburos y promover el crecimiento económico y, sobre todo, restituir los derechos de las familias nicaragüenses y lograr la reducción de la pobreza y la desigualdad.

El Programa de Inversión Pública es el principal instrumento de la política fiscal que complementa los esfuerzos productivos y sociales de largo plazo, en donde se prioriza el desarrollo de infraestructura productiva 
en sectores capaces de movilizar la actividad económica para crear empleo, priorizando el fortalecimiento de la pequeña y mediana empresa. El 69.0 por ciento de la inversión pública estará asignado a energía, transporte, agua, alcantarillado y saneamiento, salud y educación.

\section{LA PROTECCIÓN DE LA MADRE TIERRA, ADAPTACIÓN ANTE EL CAMBIO CLIMÁTICO Y GESTIÓN INTEGRAL DE RIESGOS ANTE DESASTRES}

Nicaragua es el primer país del mundo en suscribir la Declaración Universal del Bien Común de la Tierra y de la Humanidad, como compromiso del Gobierno y del país de heredar a las futuras generaciones de nicaragüenses un ambiente saludable que permita el desarrollo humano sostenible, fortaleciendo los principios, prácticas, valores y capacidades con y en beneficio de la población nicaragüense mediante la Protección de la Madre Tierra, Adaptación ante el Cambio Climático y Gestión Integral de riesgos ante desastres en el modelo del Poder Ciudadano.

El PNDH 2012-2016, es el marco estratégico nacional de políticas para continuar construyendo el Desarrollo Sostenible del país, del cual se desprenden las políticas que buscan atender los principales problemas ambientales actuales, tales como la falta de educación ambiental, la dependencia de combustible fósil, el mal manejo de cuencas hidrográficas, la contaminación del suelo, la contaminación ambiental por desechos sólidos y líquidos, el uso continuo de plaguicidas catalogados como prohibidos, el manejo inadecuado de los bosques, la pérdida de diversidad genética, la pérdida de bosques de pinares, la deforestación, el cambio climático y sus impactos, la pérdida de especies marinas y problemas sociales que aumentan los riesgos en el pueblo, como la frágil infraestructura urbana y rural y el nivel de pobreza, así como las limitaciones de recursos de nuestro país.

Se impulsaran actividades como:

-Cruzada Nacional de Reforestación

-Gestión Integral de Riesgo ante Desastres

-Gestión Integral de Riesgos

\section{Conclusiones y Discusión}

En este plan se pretende lograr que la población sepa que lo que suceda en un área tendrá repercusiones en la demás, es decir lo que suceda en el área económica tendrá implicaciones en el área social.

Otro aspecto a tomar en cuenta es que parte de bondades del PNDH es que esta en constantes cambios y en ajustes, es una expresión de estructura de modelos, es decir anteriormente los planes de desarrollo humano esta enfocados con modelos neoliberales, en donde el centro era el mercado, impulsando los tratados de libres de comercio, sin elaboración desde una estructura nacional desde las bases.

Parte de la visión que se tienen con la implementación del PNDH es transformar la vida de la gente para vivir mejor, paso a paso, poco a poco, con estos doce lineamientos y una profunda cohesión social, con una democracia participativa entre todos los actores sociales y tomadores de decisiones en busca de la mejoría.

Hemos visto grandes inversiones tanto del Gobierno como del sector privado nicaragüense para mejorar la capacidad de generación y transmisión de energía, a lo largo de los últimos años. También se ha avanzado mucho en la construcción y reparación de carreteras, principalmente de aquellas que conllevan a zonas productivas. Ambos sectores continúan siendo claves para acelerar el crecimiento económico de nuestra querida Nicaragua.

En este sentido, parte de la estrategia se focaliza en aquellos sectores donde puedan lograrse mayores impactos, y que a su vez contribuyan al logro de las metas del Plan Nacional de Desarrollo Humano de Nicaragua 2012-2016. Adicionalmente, la experiencia y el conocimiento sectorial de parte del gobierno, así como la coordinación con organismos y países donantes, son elementos que contribuyen al PNDH.

El criterio de éxito en este PNDH es el crecimiento económico con generación de trabajo y superación de la pobreza, la desigualdad, así como la eliminación del hambre. Se busca crear condiciones que faciliten la 
plena realización de las y los nicaragüenses en un país soberano e independiente, en unión con los hermanos centroamericanos, caribeños y latinoamericanos. Esto es un proceso de cambio progresivo en la calidad de vida del ser humano. Dicho proceso deberá ser sostenible, utilizando a los recursos naturales con sabiduría y protegiendo el medio ambiente, para garantizar el bienestar de las generaciones futuras y la vida en la tierra.

Desde nuestro punto de vista este plan es más sensible que cualquier otro que se haya formulado y aplicado en la historia de nuestro país, hay un interés en que la población se sienta bien es decir, el crecimiento económico, la distribución de la riqueza debe estar dirigido a las mayorías que son los más desprotegidos en nuestro país, todo lo que se haga en este plan colocar como fin, como centro a la comunidad, la familia, la restitución de los derechos.

Podría concluir que este plan incluye la voluntad del GRUN de impulsar el desarrollo, un PNDH que está en constantes cambios y en ajustes, hay parámetros de medición que año con año el gobierno utiliza para saber el avance conforme a los lineamientos planteados y que por razones como estas y como todo lo arriba descrito desde mi humilde opinión digo que este plan es sostenible, abarca los nuevos enfoques de las dimensiones del Desarrollo Sostenible, además de ser un plan con enfoques en mejorar la calidad de vida de los y las nicaragüenses en donde lo coloca como centro y sujeto primordial del desarrollo, por medio del crecimiento económico con equidad social y la transformación de los métodos de producción y de los patrones de consumo y que se sustenta en el equilibrio ecológico y el soporte vital de la región.

Podemos concluir que el presente estudio hace relevancia a los ejes dinámicos del PLAN demostrando las bondades para mejorar las condiciones de vida para los ciudadanos. Molina,

C. G., Torres, E. L., \& Urrutia, M. (1992) analizó similarmente estas condiciones en Colombia, igualmente Murgueito, R., González de Molina Navarro, M., Viglizzo, E., de Moraes, G., Dantas, M., Puignau, J. P., \& Figueroa, B. (1995) en Uruguay, por su parte Concha, A. (2002), hizo un análisis del impacto social y económico de la violencia en las Américas. Cornally, J., Crowley, T., \& ONeill (2004),

Cornally, J., Crowley, T., \& ONeill. S.(2004), lo hace en Honduras pero focalizado al análisis de las medidas para reducir la pobreza, de tal manera que podemos concluir que efectivamente los 12 lineamientos anteriormente especificados marcan una línea de bondades encaminada a un desarrollo humano. Por consiguiente en Nicaragua se ha realizado estudios que evidencian estas bondades. Franklin, H., Parra Duhalde, J., Quiroga, R., Olivera, R., Carreño, L., \& Cofre, J. (2013) analizaron el impacto ambiental, de Nicaragua, G. (2008; 2009), que contribuyo al análisis del borrador del PLAN del GRUN, Olivares, D. L. (2006), analizó el modelo turístico de carácter sistémico e integrado como facilitado del desarrollo en los países centroamericano, en la caso de Nicaragua se estudiaron los departamentos de Estelí, Nueva Segovia, Roustan-Espinosa, I. M., Quezada, F., \& Huete-Pérez, J. A. (2006) analizó las tendencias de la Biotecnología en Nicaragua, Zuniga Gonzales, CA, 2005, 2013) ha analizado los sistemas de producción agropecuarios, y la deforestación como efecto negativo en los hogares rurales como efectos del modelo de desarrollo sostenible.

Otros autores han hecho énfasis en la planificación como parte de los mecanismos para mejorar las condiciones de vida en una sociedad determinada, Vicent, M. A. H. (1998) y Vélez, I. (2003). Rice, F. P. (1997) y Sepúlveda, S. (2002) lo plantean en base al desarrollo humano y desarrollo sostenible micro regional, en tal sentido podemos afirmar que las bondades del PLAN del GRUN sobre pasa las expectativas de estos autores cuando se abordan integralmente las variables sociales, económicas, políticas y ambientales como un modelo familiar de desarrollo humano.

\section{Referencias Bibliográficas}

Arrien, J. (2006). La alfabetización en Nicaragua. Background paper prepared for the Education for All Global Monitoring Report.

Agosin, M. R., \& Machado, R. (2004). Reforma tributaria y desarrollo humano en Centroamérica. RECAUDAR PARA CRECER, 1. 
Borraz, F., \& González, N. (2008). PANES: Focalización e impacto. Revista de Ciencias Empresariales y Economía, (7), 73-110.

Concha, A. (2002). Impacto social y económico de la violencia en las Américas. Biomédica, 22, 347- 61.

Cornally, J., Crowley, T., \& ONeill, S. (2004). El Impacto de las Estrategias de Reducción de la Pobreza en el Sector Rural en Honduras y Nicaragua. Consultoría realizada por Trócaire para el Fondo Internacional de Desarrollo Agrícola (FIDA), Tegucigalpa, mayo.

de Nicaragua, G. (2008). Plan nacional de desarrollo humano 2008-2012. Documento borrador-para discusión. Abril. de Nicaragua, G. (2009). Plan nacional de desarrollo humano actualizado. Resumen Técnico.

Franklin, H., Parra Duhalde, J., Quiroga, R., Olivera, R., Carreño, L., \& Cofre, J. (2013). Nicaragua: análisis ambiental de país.

GRUN (2013). RETO para la Educación en Nicaragua 2013: construir calidad educativa, basada en cooperación genuina. MINED Gobierno de Nicaragua.

GRUN (2012). Plan Nacional de Desarrollo Humano de Nicaragua 2012-2016. Gobierno de Nicaragua.

GRUN (2011). Plan estratégico de educación 2011-2015. MINED Gobierno de Nicaragua.

Molina, C. G., Torres, E. L., \& Urrutia, M. (1992). Un plan de desarrollo humano de largo plazo para Colombia. FEDESARROLLO.

Murgueito, R., González de Molina Navarro, M., Viglizzo, E., de Moraes, G., Dantas, M., Puignau, J. P., \& Figueroa, B. (1995). Sistemas sostenibles de producción agropecuaria para campesinos (No. GTZ-1776). IICA, Montevideo (Uruguay). Programa Cooperativo para el Desarrollo Tecnológico Agropecuario del Cono Sur PROCISUR.

Olivares, D. L. (2006). El modelo turístico de carácter sistémico e integrado como facilitador del desarrollo en los países centroamericanos: el caso de Nicaragua (Estelí-Nueva Segovia). Documents d'anàlisi geogràfica, (47), 69-91.

Roustan-Espinosa, I. M., Quezada, F., \& Huete-Pérez, J. A. (2006). Tendencias actuales de la biotecnología en Nicaragua. Encuentro: Revista Académica de la Universidad Centroamericana, (75), 9-19.

Rice, F. P. (1997). Desarrollo humano: estudio del ciclo vital. Pearson Educación.

Sepúlveda, S. (2002). Desarrollo Sostenible Microregional: métodos para la planificación local. Agroamerica.

Vicent, M. A. H. (1998). Desarrollo, planificación y medio ambiente en Baja California Sur. Universidad Autónoma de Baja California.

Vélez, I. (2003). Nicaragua: el Instituto de Desarrollo Rural (IDR). En: La pobreza rural en América Latina: lecciones para una reorientación de las políticas-LC/L. 1941-P-2003-p. 159-181.

Zúniga-González, Carlos Alberto. (2013). Impacto de los Sistemas de Producción Agropecuarios en el Desarrollo Local Sostenible de Nicaragua, 1998-2005: Índice de Malmquist DEA con un Producto Orientado. Universitas (León): Revista Científica de la UNAN León, 4 (1), 10-17.

Zúniga-González, Carlos Alberto (2005). Impacto de la deforestación en el desarrollo local sostenible de los hogares: caso de Nicaragua. Encuentro No 88, pp 101-119. 